\title{
EFEITO DE UM PROGRAMA DE 35 SEMANAS DE TREINAMENTO DE FUTEBOL BASEADO NA GAME BASED APPROACH SOBRE A CAPACIDADE FÍSICA E ATENCIONAL DE CRIANÇAS E ADOLESCENTES ${ }^{i}$
}

\author{
Deborah T. Gonet, \\ Julia Castellano M., \\ Fabrício V. A. Vasconcellos ${ }^{\mathrm{ii}}$ \\ Universidade do Estado do Rio de Janeiro, \\ Rio de Janeiro, Brasil
}

\section{Resumo:}

Nos últimos anos tem crescido o interesse na utilização de uma abordagem baseada em jogos ou Game Based Approach (GBA) para ensinar e treinar futebol. Neste contexto, recorre-se aos jogos reduzidos e condicionados para promover o desenvolvimento dos praticantes. Porém, ainda são poucos os estudos que investigaram o efeito da GBA na capacidade física e especialmente em aspectos atencionais. Sendo assim, o presente estudo teve como objetivo verificar o efeito de um programa de 35 semanas de treinamento de futebol baseado na GBA sobre a capacidade cardiorrespiratória, potência muscular de membros inferiores e capacidade atencional de crianças e adolescentes. Para análise estatística comparou-se as variáveis antes e após a intervenção através do teste $t$ pareado e do Wilcoxon, adotando nível de significância de $p<0,05$. Os resultados demonstraram que houve manutenção do $\mathrm{VO}_{2}$ máximo, aumento da potência muscular de membros inferiores $(748 \pm 205 \mathrm{~W}$ vs $1250 \pm 607 \mathrm{~W}$, pré e pós, respectivamente; $\mathrm{p}=0,001)$, diminuição do tempo de reação (pré $=707 \pm 124 \mathrm{~ms}$ vs pós $=665 \pm 140 \mathrm{~ms} ; \mathrm{p}=0,023$ ) e aumento no número de acertos ( $p r e ́=81 \pm 20$ vs pós $=87 \pm 16 ; p=0,041$ ) no teste de Flanker. Dessa forma, pode-se concluir que aulas baseadas na GBA podem ser uma alternativa para melhorar a capacidade física e atencional de crianças e adolescentes.

Palavras-chave: educação física e treinamento, futebol, saúde da criança

\footnotetext{
Abstract:

In recent years, the interest of coaches and researchers about Game Based Approach (GBA) to teach and coach soccer has grown. Is this context, small-sided and conditioned

i EFFECT OF A 35 WEEK SOCCER TRAINING PROGRAM BASED ON GAME BASED APPROACH ON THE PHYSICAL AND ATTENTIONAL CAPACITY OF CHILDREN AND ADOLESCENTS

ii Correspondence: email fabricio.vasconcellos@uerj.br
} 
games are being increasingly used as a tool for player's development. However, few studies have investigated the effect of GBA on physical capacity and especially on attentional aspects. Thus, the present study aimed to verify the effect of 35-week GBA soccer training program on cardiorespiratory fitness, lower limb muscle power, reaction time and visual attention of children and adolescents. For statistical analysis, variables were compared before and after the intervention using the paired $t$ test and Wilcoxon, and the significance level adopted was $p<0.05$. The results showed a maintenance of maximum $\mathrm{VO}_{2}$ and increased lower limb muscle power $(748 \pm 205 \mathrm{~W}$ vs $1250 \pm 607 \mathrm{~W}$, before and after, respectively; $\mathrm{p}=0.001)$. In attentional capacity, a decreased in reaction time (before $=707 \pm 124 \mathrm{~ms}$ vs after $=665 \pm 140 \mathrm{~ms} ; \mathrm{p}=0.023$ ), and an increased number of correct answers (before $=81 \pm 20$ vs after $=87 \pm 16 ; p=0.041$ ) in Flanker Test. The study concluded that GBA, lasting 35 weeks, can be an alternative to improve children's physical and attentional capacity performance.

Keywords: physical education and training, soccer, child health

\section{Introdução}

Tradicionalmente os jogos esportivos coletivos são ensinados através de uma abordagem reducionista, a qual defende que através do desenvolvimento das partes é possível melhorar o todo (Da Costa; Greco; Mesquita; Garganta, 2010). No entanto, a partir da década de 80 algumas questões foram levantadas a respeito do ensino dos esportes por meio da aquisição dos fundamentos técnicos, chamando atenção para a falta de relação com o jogo (Thorpe; Bunker, 1982). Assim, a literatura sugere que através de uma abordagem baseada em jogos ou Game Based Approach (GBA) é possível proporcionar a melhoria do conhecimento e das habilidades dos indivíduos em um contexto mais específico (Serra-Olivares; González-Víllora; García-López; Araújo, 2015).

Desse modo, levando em consideração o desenvolvimento integral dos praticantes, a GBA recorre à utilização de jogos reduzidos e condicionados (JRC's) (Jarrett; Light, 2019). Os JRC's são representações do jogo formal em tamanhos menores, podendo apresentar regras modificadas (Ometto; Vasconcellos; Cunha; Teoldo et al., 2018). Além da utilização dos JRC's, a aplicação de GBA baseia-se na prática de questionamento por parte do professor (Harvey; Light, 2015), buscando promover maior capacidade de tomar decisões para resolver problemas (Clemente, 2012), maior interesse e prazer pelo esporte (Alcalá; Garijo, 2017) e o aprimoramento das habilidades específicas (Aguiar; Botelho; Lago; Maças et al., 2012). No que diz respeito ao futebol, esta abordagem pode ter um papel relevante, visto que este esporte apresenta características que aumentam a importância da dimensão informacional para a prática do jogo (Garganta; Gréhaigne, 1999; Scaglia, 2011).

Neste sentido, alguns estudos já foram desenvolvidos na tentativa de demonstrar os benefícios da utilização da GBA no ensino do futebol. Alguns dos resultados encontrados apontaram para a melhora da performance tática, tomada de decisão e 
aumento na quantidade de ações táticas realizadas no jogo de futebol (Harvey; Cushion; Wegis; Massa-Gonzalez, 2010; Osman, 2017; Souza; Müller; Costa; Graça, 2014). Contudo, em função da sua prática ser conduzida através de jogos reduzidos e condicionados, a GBA também parece ser importante para a manutenção e desenvolvimento de outros aspectos relacionados a saúde física dos praticantes.

A revisão sistemática de Aguiar e colaboradores (Aguiar; Botelho; Lago; Maças et al., 2012) demostrou que a intensidade nos jogos reduzidos é classificada como vigorosa, uma vez que a faixa da frequência cardíaca máxima (FCmáx) varia de acordo com o tamanho do campo, com valores de 75 a 87\%, podendo chegar até a 95\% (Owen; Wong; Mckenna; Dellal, 2011). Além disso, este tipo de jogo provoca grande quantidade de estímulos de mudança de direção, aceleração e desaceleração, o que também pode contribuir para o desenvolvimento da força muscular (Aguiar; Botelho; Lago; Macas et al., 2012). Dessa forma, levando em consideração a potencialidade dos JRC's em proporcionar atividades em alta intensidade e com estímulos de força, estes parecem ser uma ferramenta para promoção de saúde dos praticantes de futebol de qualquer faixa etária, com ou sem comorbidades (Vasconcellos; Seabra; Cunha; Montenegro et al., 2016).

Acrescentado a isso, na aplicação da GBA os jogadores são constantemente incentivados a pensar a respeito das ações que estão realizando e o porquê delas (Kirk; Macphail, 2002), aprimorando a atenção e as decisões tomadas pelos mesmos (Balakrishnan; Rengasamy; Aman, 2011; Osman, 2017). Devido às características do futebol, que o tornam imprevisível e aleatório, é natural que envolva uma componente de atenção elevada (Filgueiras, 2010). Esta relação entre a prática de futebol e o desenvolvimento da capacidade atencional é de extrema importância tendo em vista que indivíduos com maior capacidade atencional tendem a ter um melhor desenvolvimento cognitivo e consequentemente, melhor qualidade de vida (Diamond, 2013).

Porém, apesar da literatura apontar possíveis benefícios para utilização da GBA para o ensino e treinamento do futebol (Souza; Müller; Costa; Graça, 2013; Harvey; Cushion; Wegis; Massa-Gonzalez, 2010), ainda é pouco aplicada no dia-a-dia da prática pedagógica. Além disso, embora existam diversos estudos que tenham demonstrado efeitos positivos da prática de JRC's nas questões físicas e técnico-táticas (Aguiar; Botelho; Lago; Macas et al., 2012), provavelmente, este é o primeiro estudo a avaliar a capacidade atencional após o período de intervenção em jovens futebolistas. Sendo assim, o objetivo do presente estudo foi verificar os efeitos de um programa de 35 semanas de treinamento de futebol baseado na GBA sobre a capacidade cardiorrespiratória, potência muscular de membros inferiores e a capacidade atencional de crianças e adolescentes de 7 a 13 anos.

\section{Materiais e Métodos}

\subsection{Participantes}

Os sujeitos da amostra foram selecionados a partir do projeto de extensão denominado Núcleo de Iniciação ao Futebol (NIF), desenvolvido pelo Laboratório de Estudos em Futebol da Universidade do Estado do Rio de Janeiro - RJ, Brasil. Como único critério de 
inclusão para participar do presente estudo foi exigido apresentar atestado médico para prática regular de exercícios; e como critério de exclusão: ter assiduidade menor que 75\% das aulas ministradas. Sendo assim, apesar da turma ser constituída por 30 alunos, apenas 17 crianças, sendo 11 meninos e 6 meninas com idade entre 7 e 13 anos, atenderam aos critérios. Antes da realização da pesquisa, todos os responsáveis assinaram o Termo de Consentimento Livre e Esclarecido (TCLE) e os menores de idade assinaram o Termo de Assentimento Livre e Esclarecido (TALE). O projeto de pesquisa foi aprovado pelo Comitê de Ética em Pesquisa local com o parecer número 68786/2018.

\subsection{Procedimentos}

Foi realizada uma pesquisa longitudinal, com duração de 35 semanas. As medidas de antropometria (estatura e massa corporal) foram verificadas apenas uma vez, sendo utilizadas somente para caracterização da amostra. As avaliações foram aplicadas no mês de março e no mês de novembro do ano de 2018. As crianças foram submetidas aos testes no mesmo dia em que tinham as aulas do Núcleo de Iniciação ao Futebol. Ao chegarem no local da aula, iam diretamente para uma sala reservada onde realizavam os testes. As avaliações foram realizadas na seguinte ordem: teste de Flanker, salto vertical e teste de Yo-Yo. A ordem de avaliação foi estabelecida desta maneira para evitar que um teste influenciasse no resultado dos demais, sendo assim, o teste de maior demanda física foi realizado por último.

\subsection{Instrumentos}

\section{A. Medidas antropométricas}

A verificação da massa corporal foi realizada em uma balança da marca Filizola® e para aferir a estatura foi utilizado um estadiômetro vertical da marca Sanny® fixo na parede. Foi calculado o índice de massa corporal (IMC) de acordo com a OMS (2007), através da divisão da massa corporal $(\mathrm{kg})$ pelo quadrado da estatura $\left(\mathrm{m}^{2}\right)$. As avaliações antropométricas seguiram o proposto pela International Society for Advancement in Kinanthropometry (ISAK).

\section{B. Capacidade cardiorrespiratória}

Foi utilizado o Yo-yo Intermittent Recovery Test Level 1 adaptado para crianças para estimar o consumo máximo de oxigênio (Ahler; Bendiksen; Krustrup; Wedderkopp, 2012). A fórmula utilizada para o cálculo do $\mathrm{VO}_{2}$ máximo foi: $V O_{2} m a ́ x(\mathrm{ml} / \mathrm{min} / \mathrm{kg})=$ distância IR1 (m) x 0,0084 + 36,4 (Bangsbo; Iaia; Krustrup, 2008). Este teste consiste em corrida de vai e vem com uma distância de 16 metros e com 4 metros reservados para os 10 segundos de descanso. O ritmo da corrida é fornecido por sinais sonoros emitidos por um aplicativo e transmitidos através de reprodutor portátil, com incremento progressivo da velocidade até a exaustão. O teste foi encerrado quando, por duas vezes, o indivíduo não conseguiu retornar à linha de partida antes do sinal sonoro, ou quando o participante se sentiu incapaz de realizar outra ida e volta na velocidade ditada. 


\section{Potência muscular de membros inferiores}

A potência de membros inferiores foi verificada por meio de uma plataforma de contato (Jump System Pro, Cefise ${ }^{\circledR}$, Nova Odessa, SP, Brasil). A criança se posicionava descalça sobre a plataforma, com os membros inferiores em extensão. Os avaliadores orientaram as crianças a manterem as mãos na altura da cintura pélvica durante o salto. Após comando verbal, a criança realizava um agachamento seguido de um salto, visando atingir a máxima altura possível, sem flexionar o joelho enquanto estivesse no ar.

Foram realizados 3 saltos com 1 minuto de intervalo entre eles e foi considerado como resultado final a maior altura e potência máxima atingida entre os três (Markovic; Dizdar; Jukic; Cardinale, 2004). A altura do salto foi calculada a partir do tempo de voo através da fórmula: $h=g \cdot t^{2} \cdot{ }^{-1}$, sendo " $h$ " a altura, "g" o valor da aceleração da gravidade $\mathrm{e}$ " $\mathrm{t}$ " o tempo de voo. A potência foi calculada pelo software através do tempo de voo do salto, ou seja, de acordo com o último momento de contato com o tapete até o primeiro contato após a realização do salto (Carneiro; Costa; Santos; Da Silva et al., 2019). Para estes cálculos foi utilizado o software Jump System Pro 1.0 da Cefise ${ }^{\circledR}$.

\section{Capacidade atencional}

A capacidade atencional foi mensurada através do teste de Flanker (Eriksen, 1995), para isso, utilizou-se um notebook Dell Inspiron (Dell Inc., Texas, EUA) com o software EPrime (Psychology Software Tools Inc, USA). Para realizar o teste, o aluno sentava em uma cadeira e ficava a $30 \mathrm{~cm}$ de distância da tela do notebook, não tendo nenhuma outra distração naquele ambiente. $\mathrm{O}$ computador apresentava cinco setas em sua tela, sendo indicado que o aluno mantivesse a atenção somente na seta central. Se a seta central apontasse para a direita, o aluno deveria aperta a tecla "L" do teclado. Caso a seta central estivesse apontada para a esquerda, tecla " $S$ ". O protocolo utilizado era composto por 100 trials, 50 deles com as cinco setas apontadas para o mesmo lado (congruentes) e 50 com as setas apontadas para lados opostos (incongruentes). Antes destes 100 trials principais, foram realizados 20 trials para familiarização. Os sujeitos foram incentivados a responder o mais rápido possível, porém com cuidado para acertar as respostas. A quantidade de acertos e o tempo de reação médio foram as duas medidas utilizadas para análise dos dados.

\section{E. Programa de treinamento}

O programa de treinos foi realizado na Universidade do Estado do Rio de Janeiro, oferecido duas vezes por semana com uma única turma às terças e quintas-feiras, com horário de início às $18 \mathrm{~h}$ e término às $19 \mathrm{~h}$. A turma era composta por 30 crianças, entre meninos e meninas, de idade entre 7 e 13 anos. As atividades aconteciam no campo de society da universidade e eram conduzidas por professores do Laboratório de Estudos em Futebol (Labesfut).

Os primeiros 10 minutos da aula eram destinados à uma conversa inicial com os alunos sobre os principais acontecimentos do meio esportivo, com destaque para os jogos e campeonatos de futebol. Em seguida a turma era dividida em dois grupos de acordo com a idade, um grupo era formado pelas crianças de 7 até 9 anos e o outro por crianças de 10 anos em diante. Os 20 minutos seguintes eram dedicados à jogos de tamanhos 
menores (1x1 até 3x3), seguidos por 20 minutos de jogos maiores (4x4 em diante). Os 10 minutos finais eram voltados à desafios com bola para os alunos, como por exemplo, acertar o travessão. Cada grupo realizava as atividades sob o comando de dois professores do projeto e utilizava a metade do campo de society.

A duração das atividades era em torno de 50 minutos e os exercícios realizados durante as aulas eram discutidos e estabelecidos pelos professores na semana anterior. As atividades ministradas nas aulas apresentavam os mesmos objetivos em ambas as categorias de idade, porém a complexidade era acrescentada ou diminuída de acordo com o nível da turma. Os jogos reduzidos e condicionados apresentados tinham as seguintes manipulações: alteração das formas de pontuação e quantidade de alvos, superioridade numérica da equipe de ataque com a utilização de jogadores de apoio e coringas, diferentes tamanhos e formatos de campo e a implementação de regras como limitação de toques na bola e restrição de tempo para finalizar a jogada.

\subsection{Análise estatística}

Utilizou-se o software SPSS 25.0 para realizar as análises estatísticas do presente estudo. A normalidade dos dados foi verificada através do teste de Kolmogorov-Smirnov. As variáveis foram comparadas antes e após a intervenção utilizando somente um grupo. Para comparar as variáveis paramétricas foi utilizado o teste $t$ pareado e para comparar as variáveis não-paramétricas utilizou-se o teste de Wilcoxon pareado. $O$ nível de significância adotado foi de $\mathrm{p}<0,05$. Para determinar o tamanho de efeito recorreu-se ao teste $d$ de Cohen. $O$ tamanho do efeito considerado foi: efeito insignificante $(d<0,19)$, efeito pequeno $(0,20<d<0,49)$, efeito médio $(0,50<d<0,79)$, efeito grande $(0,80<d<1,29)$ e efeito muito grande $(d>1,30)$ (Rosenthal, 1996).

\section{Resultados}

O presente estudo contou com a participação de 17 crianças com idades entre 7 e 13 anos, de ambos os sexos, inscritas no projeto de extensão intitulado Núcleo de Iniciação ao Futebol da UERJ. A tabela 1 apresenta os dados descritivos com média e desvio padrão das variáveis. Conforme apresentado, identifica-se que o valor médio de IMC apresentado pelos alunos é considerado pela OMS (2011) como um perfil eutrófico.

Tabela 1: Estatística descritiva das características antropométricas da amostra $(\mathrm{n}=17)$

\begin{tabular}{lcc}
\hline Variáveis & Média & Desvio Padrão \\
\hline Idade (anos) & 9,35 & 1,54 \\
Estatura $(\mathrm{m})$ & 1,40 & 12,29 \\
Massa corporal $(\mathrm{kg})$ & 38,63 & 0,10 \\
$\mathrm{IMC}\left(\mathrm{kg} / \mathrm{m}^{2}\right)$ & 19,29 & 4,28 \\
\hline
\end{tabular}

Nota: IMC - índice de massa corporal 
Sobre o efeito do treinamento na capacidade cardiorrespiratória dos indivíduos, verifica-se que apesar de apresentar um pequeno aumento do $\mathrm{VO}_{2}$ máximo, não houve diferença entre as medidas obtidas antes e após a intervenção (Figura 1a). Diferentemente da capacidade cardiorrespiratória, a potência de membros inferiores aumentou significativamente após as semanas de treinamento $(d=0,85$; efeito grande). Como pode ser visto na figura 1.b, houve aumento de aproximadamente $67 \%$ da potência muscular após o treinamento baseado na GBA.

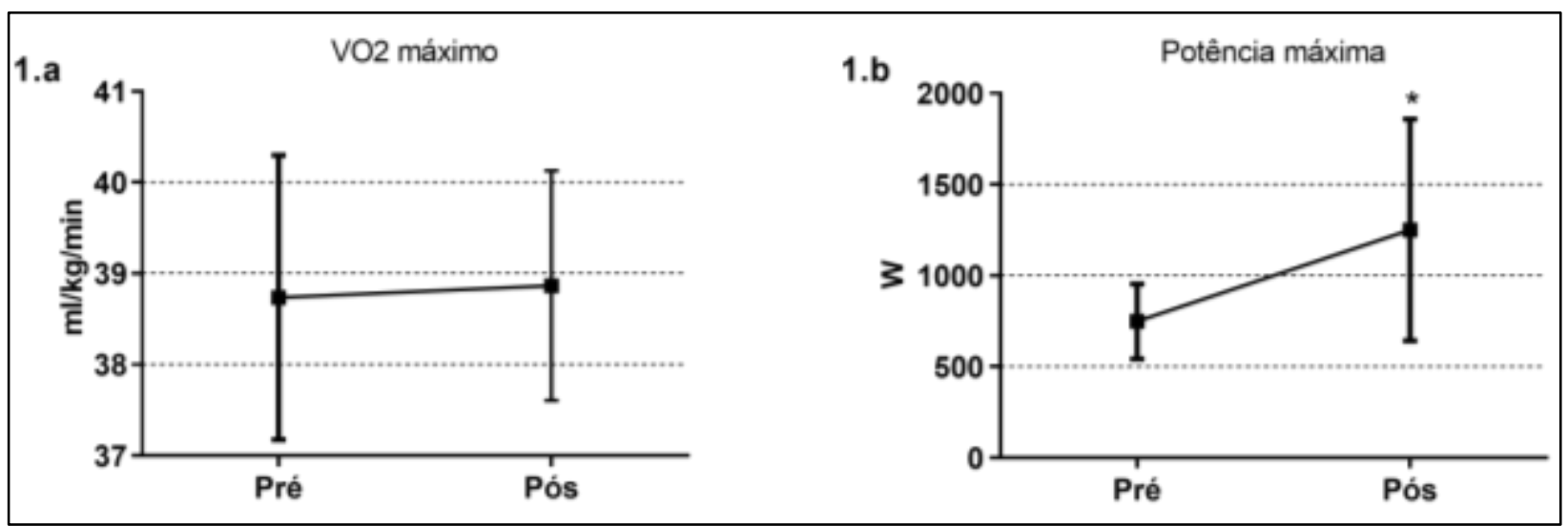

Figura 1: Comparação das variáveis de saúde pré e pós intervenção

Nota: 1.a - Comparação do $\mathrm{VO}_{2}$ máximo pré e pós intervenção. 1.b - Comparação da potência máxima de membros inferiores pré e pós intervenção. *diferença significativa entre pré e pós intervenção $(p<0,05)$

No que se refere à capacidade atencional, avaliada através do teste de Flanker, houve aumento da quantidade de acertos após as 35 semanas de treinamento de futebol baseado na GBA ( $d=0,24$; efeito pequeno), como pode ser observado na figura $2 \mathrm{a}$. Assim como o tempo de reação médio, que diminuiu significativamente após o programa de treinamento, o que pode ser observado na figura $2 b(d=0,26$; efeito pequeno).

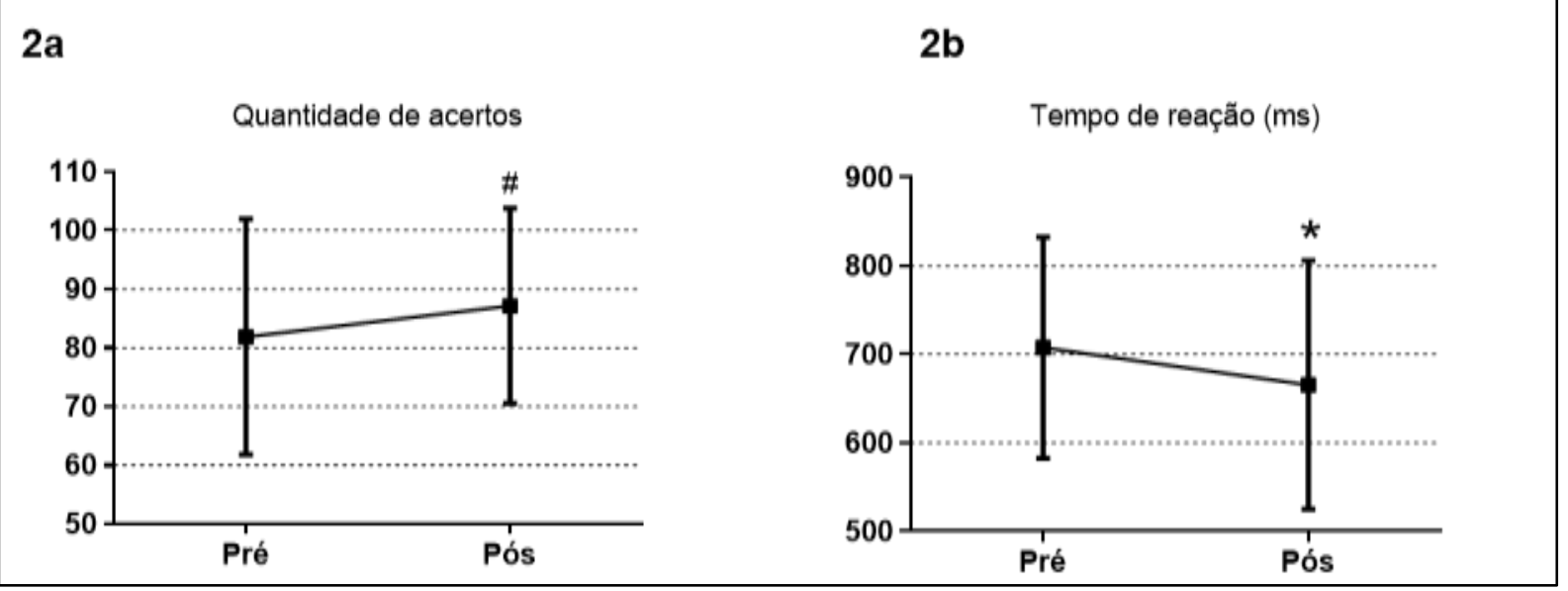

Figura 2: Comparação da capacidade atencional pré e pós intervenção

Nota: 2.a - Quantidade de acertos no Teste de Flanker. 2.b - Tempo de reação médio no Teste de Flanker. $\# \mathrm{p}=0,041^{*} \mathrm{p}=0,023$ 


\section{Discussão}

O presente estudo teve como objetivo verificar o efeito do treinamento de futebol baseado na Game Based Approach sobre a potência muscular de membros inferiores e capacidade cardiorrespiratória e atencional de meninos e meninas de 7 a 13 anos de idade. Os resultados do estudo mostraram que as crianças e adolescentes apresentaram melhora significativa da potência muscular de membros inferiores e da atenção visual.

Embora a aptidão cardiorrespiratória seja um forte preditor de saúde em idade pediátrica (Rech; Halpern; Costanzi; Bergmann et al., 2010) e o presente estudo não tenha demonstrado melhora nesta condição, este resultado está alinhando a outros encontrados na literatura como o estudo de Faude (2010), o qual não apontou diferença nos valores de $\mathrm{VO}_{2}$ após seis meses de intervenção com futebol recreativo para jovens obesos. Assim, a aptidão cardiorrespiratória da amostra do presente estudo foi classificada como baixa antes e após a intervenção (Venâncio; Ferreira; Teixeira; Fernandes et al., 2012), porém isso pode ser explicado segundo as recomendações da Organização Mundial da Saúde, que orienta que crianças acumulem pelo menos 60 minutos/dia de atividade física moderada a vigorosa para melhorar dentre outras capacidades, a aptidão cardiorrespiratória (World Health, 2010), e no presente estudo o volume acumulado semanal de treino foi insuficiente ( 120 minutos/semana).

Apesar disso, os JRC's favorecem a participação dos indivíduos no jogo, aumentando assim a quantidade de ações com bola, como passes e chutes, habilidades estas que estimulam o desenvolvimento da força da musculatura dos membros inferiores (Aguiar; Botelho; Lago; Macas et al., 2012). Ademais, os JRC's promovem constantes mudanças de direção, devido ao tamanho reduzido dos campos utilizados, elevando a frequência de contrações excêntricas dos músculos dos membros inferiores, favorecendo o aumento da potência muscular (Hill-Haas; Dawson; Impellizzeri; Coutts, 2011). Vale ressaltar que a força muscular tem um papel de destaque como um importante marcador de saúde, sendo associada com um perfil cardiovascular saudável em crianças e adolescentes (Fernandez-Santos; Ruiz; Cohen; Gonzalez-Montesinos et al., 2015).

Embora o presente estudo não tenha avaliado o nível maturacional dos sujeitos da amostra, a idade média dos indivíduos sugere que eles ainda não estavam passando pelo processo de maturação biológica, pois sabe-se que o pico de velocidade do crescimento (PVC) tem ocorrência entre 12 e 16 anos de idade em meninos e entre 10 e 14 anos em meninas, e que além disso, a elevação da produção dos hormônios só acontece após o PVC (RÉ, 2011). Ademais, uma melhora do desempenho da potência muscular em decorrência da maturação, provavelmente, também representaria em melhora do desempenho cardiovascular, fato que não acorreu no presente estudo. Dessa forma, acredita-se que a aplicação da GBA, que tem por característica ser baseado na utilização de JRC's, foi o principal motivo do aumento significativo da potência muscular de membros inferiores das crianças.

Além disso, através da avaliação da capacidade atencional por meio do teste de Flanker foi possível aferir os processos de atenção visual (ERIKSEN, 1995), dando 
destaque para a mensuração de menor tempo de reação após o treinamento de futebol baseado na GBA. Neste sentido, acredita-se que a utilização de jogos com tamanho reduzido tenha promovido melhora do tempo de reação, pois esta manipulação promove maior proximidade entre as crianças durante os jogos, estimulando que encontrem soluções mais rapidamente para os problemas encontrados.

Assim como, a estratégia de oportunizar a prática do jogo em detrimento da execução do gesto técnico isolado faz com que os praticantes sejam constantemente incentivados a pensar a respeito das ações que estão fazendo e o porquê delas (Kirk; Macphail, 2002). Desse modo, auxilia no desenvolvimento da atenção visual e contribui com a capacidade de tomar decisões (Balakrishnan; Rengasamy; Aman, 2011; Osman, 2017), fato este que ajuda a explicar os resultados encontrados a respeito da quantidade de acertos. O desenvolvimento da capacidade atencional demonstrado pelo teste de Flanker surge como um benefício importante da aplicação da GBA visto que a literatura aponta que indivíduos com melhor capacidade cognitiva tornam-se mais bem preparados em muitas outras áreas da vida, como na saúde física, desempenho escolar, sucesso no trabalho e harmonia matrimonial (Diamond, 2013).

Embora tenham sido apresentados resultados importantes em relação aos benefícios do treinamento de futebol baseado na Game Based Approach sob aspectos atencionais e a capacidade física, cabe reconhecer que o presente estudo apresenta como limitação a falta de um grupo controle para comparar com o grupo experimental, fato que faz com que seja necessária uma interpretação cuidadosa dos resultados. Neste sentido, é necessário que mais estudos sejam realizados nesta área, procurando identificar os possíveis benefícios que o treinamento esportivo baseado na GBA é capaz de proporcionar aos indivíduos expostos à essa abordagem.

\section{Conclusão}

Os resultados encontrados apontam que um programa de treinamento de futebol baseado na Game Based Approach pode promover o desenvolvimento da potência muscular de membros inferiores e da capacidade atencional de crianças e adolescentes. Dessa forma, no que diz respeito ao ensino e treinamento dos esportes, professores e treinadores podem recorrer à esta abordagem, tendo em vista que sua aplicação vai além das ações técnico-táticas, sendo eficaz também na estimulação da capacidade atencional e no desempenho físico.

\section{Conflitos de interesse}

Os autores declaram que não há conflitos de interesses em relação ao artigo.

\section{Sobre os autores}

Deborah T. Gonet possui graduação em Licenciatura (2018) e está cursando Bacharel em Educação Física na Universidade do Estado do Rio de Janeiro (UERJ). Participante do 
Laboratório de Estudos em Futebol (LABESFUT-UERJ) e mestranda no Programa de PósGraduação em Ciências do Exercício e do Esporte (PPGCEE-UERJ).

Julia C. Marques possui graduação em Bacharelado (2018) e está cursando Licenciatura em Educação Física pela Universidade do Estado do Rio de Janeiro (UERJ). Participante do Laboratório de Estudos em Futebol (LABESFUT-UERJ) e mestranda no Programa de Pós-Graduação em Ciências do Exercício e do Esporte (PPGCEE-UERJ).

Fabrício V. A. Vasconcellos possui graduação em Licenciatura plena em Educação Física pela Universidade do Estado do Rio de Janeiro (2005), Pós-graduação em Treinamento Desportivo pela Universidade gama Filho (2007), Mestrado em Ciência da Motricidade Humana pela Universidade Castelo Branco (2009) e Doutorado em Ciências do Desporto pela Universidade do Porto (2014). Atualmente é professor adjunto da disciplina de Futebol de Campo do Instituto de Educação Física e Desportos da Universidade do Estado do Rio de Janeiro, Coordenador do Laboratório de Estudos em Futebol (LABESFUT) da Universidade do Estado do Rio de Janeiro e Docente Permanente do Programa de Pós-Graduação em Ciências do Exercício e do Esporte (PPGCEE/UERJ).

Laboratório de Estudos em Futebol (LABESFUT) - www.labesfut.com.br

\section{Agradecimentos}

O presente trabalho foi realizado com apoio da Coordenação de Aperfeiçoamento de Pessoal de Nível Superior - Brasil (CAPES) - Código de Financiamento 001.

\section{Referências}

Aguiar, M.; Botelho, G.; Lago, C.; Maças, V. et al. A review on the effects of soccer smallsided games. Journal of human kinetics, 33, p. 103-113, 2012. doi: 10.2478/v10078012-0049-x

Ahler, T.; Bendiksen, M.; Krustrup, P.; Wedderkopp, N. Aerobic fitness testing in 6-to 9year-old children: reliability and validity of a modified Yo-Yo IR1 test and the Andersen test. European journal of applied physiology, 112, n. 3, p. 871-876, 2012. doi: 10.1007/s00421-011-2039-4

Alcalá, D. H.; Garijo, A. H. Teaching Games for Understanding: A Comprehensive Approach to Promote Student's Motivation in Physical Education. Journal of human kinetics, 59, n. 1, p. 17-27, 2017. doi: 10.1515/hukin-2017-0144

Balakrishnan, M.; Rengasamy, S.; Aman, M. S. Effect of teaching games for understanding approach on students' cognitive learning outcome. International Journal of Social, Behavioral, Educational, Economic, Business and Industrial Engineering, 5, n. 5, p.714-716, 2011. doi: 10.5281/zenodo.1074670

Bangsbo, J.; Iaia, F. M.; Krustrup, P. The Yo-Yo intermittent recovery test. Sports medicine, 38, n. 1, p. 37-51, 2008. doi: 10.2165/00007256-200838010-00004 
Carneiro, T.; Costa, O.; Santos, W.; Da Silva, W. et al. Acompañamiento de la potencia de miembros inferiores en jóvenes jugadores de fútbol. Revista Peruana de ciencia de la actividad fisica y del deporte, 6, n. 2, p. 10-10, 2019.

Clemente, F. M. Princípios pedagógicos dos teaching games for understanding e da pedagogia não-linear no ensino da educação física. Movimento, 18, n. 2, 2012. doi: 10.22456/1982-8918.27495

Da Costa, I. T.; Greco, P. J.; Mesquita, I.; Garganta, J. O Teaching Games for Understanding (TGfU) como modelo de ensino dos jogos desportivos coletivos. Revista Palestra, 10, p. 69-77, 2010.

Diamond, A. Executive functions. Annual review of psychology, 64, p. 135-168, 2013. doi: 10.1146/annurev-psych-113011-143750

Eriksen, C. W. The flankers task and response competition: A useful tool for investigating a variety of cognitive problems. Visual Cognition, 2, n. 2-3, p. 101-118, 1995. doi: $10.1080 / 13506289508401726$

Faude, O.; Kerper, O.; Multhaupt, M.; Winter, C. et al. Football to tackle overweight in children. Scandinavian journal of medicine \& science in sports, 20, n. s1, p. 103110, 2010. doi: 10.1111/j.1600-0838.2009.01087.x

Fernandez-Santos, J. R.; Ruiz, J. R.; Cohen, D. D.; Gonzalez-Montesinos, J. L. et al. Reliability and validity of tests to assess lower-body muscular power in children. The Journal of Strength \& Conditioning Research, 29, n. 8, p. 2277-2285, 2015. doi: 10.1519/JSC.0000000000000864

Filgueiras, A. Abordagem neuropsicológica dos processos de orientação da atenção visuo-espacial e manutenção da concentração em atletas da categoria sub-13 de futebol de campo. Ciências \& Cognição, 15, n. 2, 2010.

Garganta, J.; Gréhaigne, J. F. Abordagem sistêmica do jogo de futebol: moda ou necessidade? Movimento (ESEFID/UFRGS), 5, n. 10, p. 40-50, 1999. doi: 10.22456/1982-8918.2457

Harvey, S.; Cushion, C. J.; Wegis, H. M.; Massa-Gonzalez, A. N. Teaching games for understanding in American high-school soccer: A quantitative data analysis using the game performance assessment instrument. Physical Education and Sport Pedagogy, 15, n. 1, p. 29-54, 2010. doi: 10.1080/17408980902729354

Harvey, S.; Light, R. L. Questioning for learning in game-based approaches to teaching and coaching. Asia-Pacific Journal of Health, Sport and Physical Education, 6, n. 2, p. 175-190, 2015. doi: 10.1080/18377122.2015.1051268

Hill-Haas, S. V.; Dawson, B.; Impellizzeri, F. M.; COUTTS, A. J. Physiology of small-sided games training in football. Sports medicine, 41, n. 3, p. 199-220, 2011. doi: 10.2165/11539740-000000000-00000

Jarrett, K.; Light, R. The experience of teaching using a game based approach: Teachers as learners, collaborators and catalysts. European Physical Education Review, 25, n. 2, p. 565-580, 2019. doi: 10.1177/1356336X17753023 
Kirk, D.; Macphail, A. Teaching games for understanding and situated learning: Rethinking the Bunker-Thorpe model. Journal of teaching in Physical Education, 21, n. 2, p. 177-192, 2002. doi: 10.1123/jtpe.21.2.177

Markovic, G.; Dizdar, D.; Jukic, I.; Cardinale, M. Reliability and factorial validity of squat and countermovement jump tests. The Journal of Strength \& Conditioning Research, 18, n. 3, p. 551-555, 2004. doi: 10.1519/15334287(2004)18<551:RAFVOS >2.0.CO;2

Ometto, L.; Vasconcellos, F. V. A.; Cunha, F. A.; Teoldo, I. et al. How manipulating task constraints in small-sided and conditioned games shapes emergence of individual and collective tactical behaviours in football: A systematic review. International Journal of Sports Science \& Coaching, p. 1747954118769183, 2018. doi: $10.1177 / 1747954118769183$

Osman, A. Effects of teaching games for understanding on tactical awareness and decision making in soccer for college students. Ovidius University Annals, Series Physical Education and Sport/Science, Movement and Health, 17, n. 2, p. 170-177, 2017.

Owen, A. L.; Wong, D. P.; Mckenna, M.; Dellal, A. Heart rate responses and technical comparison between small-vs. large-sided games in elite professional soccer. The Journal of Strength \& Conditioning Research, 25, n. 8, p. 2104-2110, 2011. doi: 10.1519/JSC.0b013e3181f0a8a

Rech, R. R.; Halpern, R.; Costanzi, C. B.; Bergmann, M. L. D. A. et al. Prevalência de obesidade em escolares de 7 a 12 anos de uma cidade Serrana do RS, Brasil. Revista Brasileira de Cineantropometria \& Desempenho Humano, 12, n. 2, p. 90-97, 2010. doi: 10.5007/1980-0037.2010v12n2p90

Rosenthal, J. A. Qualitative descriptors of strength of association and effect size. Journal of social service Research, 21, n. 4, p. 37-59, 1996. doi: 10.1300/J079v21n04_02

Ré, A. H. N. Growth, maturation and development during childhood and adolescence: Implications for sports practice. Motricidade, 7, n. 3, p. 55-67, 2011. doi: 10.6063/motricidade.7(3).103

Scaglia, A. J. O futebol e as brincadeiras de bola: a família dos jogos de bola com os pés. Phorte, 2011.

Serra-Olivares, J.; González-Víllora, S.; García-López, L. M.; ARAÚJO, D. Game-based approaches' pedagogical principles: Exploring task constraints in youth soccer. Journal of human kinetics, 46, n. 1, p. 251-261, 2015. doi: 10.1515/hukin-2015-0053

Souza, C. R. B. C. D.; Müller, E. S.; Costa, I. T.; Graça, A. B. S. Quais comportamentos táticos de jogadores de futebol da categoria sub-14 podem melhorar após 20 sessões de treino? Revista Brasileira de Ciências do Esporte, 36, n. 1, p. 71-86, 2014. doi: 10.1590/S0101-32892014000100006

Thorpe, R.; Bunker, D. From theory to practice: two examples of an understanding approach to the teaching of games. Bulletin of Physical Education, 18, n. 1, p. 9-15, 1982. 
Vasconcellos, F.; Seabra, A.; Cunha, F.; Montenegro, R. et al. Health markers in obese adolescents improved by a 12-week recreational soccer program: a randomised controlled trial. Journal of sports sciences, 34, n. 6, p. 564-575, 2016. doi: 10.1080/02640414.2015.1064150

Venâncio, P. E. M.; Ferreira, C. E. S.; Teixeira, C. G. O.; Fernandes, R. M. et al. Aptidão cardiorrespirátoria em diferentes modalidades esportivas de crianças e adolescentes da cidade de Anápolis, GO. Motricidade, 8, n. 2, p. 549-554, 2012.

World Health, O. Global recommendations on physical activity for health. World Health Organization, 2010.

World Health, O. Obesity and Overweight. Geneva: World Health Organization; 2012. 2011. 
Deborah T. Gonet, Julia Castellano, Fabrício V. A. Vasconcellos

EFEITO DE UM PROGRAMA DE 35 SEMANAS DE TREINAMENTO DE FUTEBOL BASEADO NA

GAME BASED APPROACH NA CAPACIDADE FÍSICA E ATENCIONAL DE CRIANÇAS E ADOLESCENTES

Creative Commons licensing terms

Authors will retain the copyright of their published articles agreeing that a Creative Commons Attribution 4.0 International License (CC BY 4.0) terms will be applied to their work. Under the terms of this license, no permission is required from the author(s) or publisher for members of the community to copy, distribute, transmit or adapt the article content, providing a proper, prominent and unambiguous attribution to the authors in a manner that makes clear that the materials are being reused under permission of a Creative Commons License. Views, opinions and conclusions expressed in this research article are views, opinions and conclusions of the author(s). Open Access Publishing Group and European Journal of Physical Education and Sport Science shall not be responsible or answerable for any loss, damage or liability caused in relation to/arising out of conflict of interests, copyright violations and inappropriate or inaccurate use of any kind content related or integrated on the research work. All the published works are meeting the Open Access Publishing requirements and can be freely accessed, shared, modified, distributed and used in educational, commercial and non-commercial purposes under a Creative Commons attribution 4.0 International License (CC BY 4.0). 